\title{
Lumbar intervertebral disc allograft transplantation: long-term mobility and impact on the adjacent segments
}

\author{
Yong-Can Huang ${ }^{\mathrm{a}, 1}$, Jun Xiao ${ }^{\mathrm{b}, 1}$, William W. Lu ${ }^{\mathrm{a}}$, Victor Y. L. Leung ${ }^{\mathrm{a}}$, Yong $\mathrm{Hu}^{\mathrm{a}}$, \\ Keith D.K. Luk ${ }^{\mathrm{a}, *}$ \\ ${ }^{a}$ Department of Orthopaedics and Traumatology, The University of Hong Kong, \\ Hong Kong SAR, China; \\ ${ }^{b}$ Department of Joint Surgery, Nanfang Hospital, Southern Medical University, \\ Guangzhou, China.
}

${ }^{1}$ Yong-Can Huang and Jun Xiao contributed equally to this work.

*To whom correspondence should be addressed: Prof. Keith D.K. Luk, Department of Orthopaedics and Traumatology, The University of Hong Kong, 5/F Professor Block, Queen Mary Hospital, Pokfulam, Hong Kong SAR, China. Tel: +852 2255 4254; Fax: +852 2817 4392; E-mail: $\underline{\text { hrmoldk@ @ku.hk }}$

Conflict of interest: None. 


\begin{abstract}
(258 words)
Purpose Fresh-frozen intervertebral disc (IVD) allograft transplantation has been successfully performed in the human cervical spine. Whether this non-fusion technology could truly decrease adjacent segment disease is still unknown. This study evaluated the long-term mobility of the IVD-transplanted segment and the impact on the adjacent spinal segments in a goat model.
\end{abstract}

Methods Twelve goats were used. IVD allograft transplantation was performed at lumbar L4/L5 in 5 goats; the other 7 goats were used as the untreated control (5) and for the supply of allografts (2). Post-operation lateral radiographs of the lumbar spine in the neutral, full flexion and full extension positions were taken at 1, 3, 6, 9 and 12 months. Disc height (DH) of the allograft and the adjacent levels was calculated and range of motion (ROM) was measured using the Cobb's method. The anatomy of the adjacent discs was observed histologically.

Results DH of the transplanted-segment was decreased significantly after 3 months but no further reduction was recorded until the final follow-up. No obvious alteration was seen in the ROM of the transplanted segment at different time points with the ROM at 12 months being comparable to that of the untreated control. The DH and ROM in the adjacent segments were well maintained during the whole observation period. At postoperative 12 months, the ROM of the adjacent levels was similar to that of the untreated control and the anatomical morphology was well preserved.

Conclusions Lumbar IVD allograft transplantation in goats could restore the segmental mobility and did not negatively affect the adjacent segments after 12 
months.

Key words: Intervertebral disc; allograft; transplantation; mobility; adjacent segments

\section{Introduction}

Spinal fusion and artificial disc replacement are the most frequently employed salvage procedures for severe intervertebral disc (IVD) degeneration [1]; they are generally effective in pain alleviation, reduction of pain medication consumption and improvement of life quality [2, 3]. As an alternative to these two surgical techniques, fresh-frozen IVD allograft transplantation has been successfully performed in the cervical spine of 13 patients, where it provided acceptable clinical outcomes for up to 10 years $[4,5]$. Although rapid degeneration of the transplanted IVD allograft was observed in some cases, the neurological symptoms, range of motion (ROM) and clinical stability of the spinal unit were well preserved [4].

In goat lumbar spine, motion preservation potential of IVD allografting has been partly validated by in-vitro three-dimensional kinematic analysis, it was reported that at 6 months postoperatively IVD allografting was able to restore segmental motion and did not change the neutral zone [6]. Nevertheless, the long-term results were not well understood. Hence, the first aim in this work is to address the sequential changes of the disc height (DH) and segmental motion of the lumbar spine after IVD allografting in goats.

After spinal fusion and artificial disc replacement, one of the late sequelae is 
adjacent segment degeneration (ASD) [7-10]. ASD represents the radiographic changes of the discs adjacent to the surgically treated segment [11], and at present its etiology remains unclear [12]. In the human cervical spine after IVD allografting, none of the adjacent segments showed evidence of accelerated degeneration at a minimum follow-up of 5 years; this advantage may be attributed to the biological remodeling potential of the allograft thus preserving the natural global and focal biomechanics of the spine [4].The natural remodeling potential was also observed in the mal-positioned IVD allograft in a goat lumbar spine experiment [6]. It was hence hypothesized that this remodeling potential of IVD allograft might help prevent the adverse changes in the adjacent segments, thus protecting them from ASD. To test this hypothesis, the second aim of this study is thus to investigate the mobility and histological changes of the cranial and caudal discs after IVD allografting in a goat lumbar spine model.

\section{Materials and methods}

\subsection{Experimental design}

In this study, using the newly-developed surgical techniques [13], fresh-frozen IVD allograft transplantation was performed in the lumbar spine (L4/L5) of 5 goats without internal fixation. DH and ROM in the IVD-transplanted segment and in the adjacent levels were measured after transplantation for 1, 3, 6, 9 and 12 months. Then DHs of the three levels at different time slots were compared with those immediately post operation, respectively; ROMs of the IVD-transplanted segment and the adjacent 
levels were calculated at different time slots and then compared with that of the untreated control group at postoperative 12 months, respectively. At the final follow-up, histological examination of the adjacent discs was conducted.

\subsection{Animals}

The research proposal has been approved by Committee on the Use of Live Animals in Teaching \& Research, the University of Hong Kong (CULATR1872-09). Twelve male goats (Hainan East Goats) between 6 and 9 months weighing between 17.5 and $25 \mathrm{~kg}$ were used in this study. Two goats served as the IVD allografts donor, 5 goats as allograft recipients and the remaining 5 untreated goats as normal controls.

\subsection{Fresh-frozen intervertebral disc allograft transplantation}

Fresh-frozen IVD allograft transplantation without internal fixation was performed at lumbar L4/L5 as previous description [13]. In short, after intravenous injection of a mixture of Ketamine $(10 \mathrm{mg} / \mathrm{kg})$ and Xylazine $(0.1 \mathrm{mg} / \mathrm{kg})$ and endotracheal intubation, the IVD was exposed using the "retro-psoas" approach. The nucleus pulposus, the cartilaginous and bony endplates and the posterior annulus fibrosus were removed completely to form a recipient slot. The fresh-frozen IVD allograft with the most compatible size was selected, thawed and thumbed into the slot with only finger pressure; no internal fixation of any form was used. The psoas muscle was then repositioned and the wound was closed routinely. In the five goats of the untreated group, lumbar disc (L4/L5) exposure was conducted using the "retro-psoas" approach and then the wound was closed routinely. All these ten goats after IVD allograft transplantation and disc exposure only were allowed free 
mobilization immediately after surgery in an open field and they were followed up with a final radiography at 12 months.

\subsection{Lateral radiography}

Digital radiography system (SIEMENS, Germany) was used for images acquisition with the exposure factor: $100 \mathrm{~mA}, 85 \mathrm{kV}$ and $500 \mathrm{~ms}$. Immediately after surgery, left lateral radiography was taken to observe the position of the IVD allograft in the 5 goats. At 1, 3, 6, 9 and 12 months after the IVD allografting, the 5 goats were fasted for about $48 \mathrm{~h}$ before lateral radiographs of the lumbar spine at the neutral, full flexion and full extension positions were taken under general anaesthesia. Radiographs at these positions were also taken in the five goats of untreated control after follow-up for 12 months. As shown in Fig.1A-B, full flexion was achieved by securing a rope above the carpus of forelimbs and the tarsus of hindlimbs by binding the four limbs together with the lumbar spine held in the maximally flexed position $[6$, 14]. For the extension radiograph, the goat was placed supine with a customized bolster placed directly under the operated segment with both forelimbs tied to one end of the table while both hindlimbs were tied to the opposite end, according to the previous literatures $[6,14]$. No standardization to the loads or displacements was applied during the radiographs collection.

\subsection{Measurement of disc allograft height}

Definition of DH of the disc allografts is an average of the intervertebral distances between the two bony endplates after transplantation as measured on the lateral plain radiographs. The average $\mathrm{DH}$ was calculated with the measurements obtained from 
the anterior, middle and posterior portions of the disc allograft and the adjacent levels according to the methods previously described $[15,16]$.

\subsection{Measurement of ROM}

ROM of the disc-transplanted segment and the adjacent levels was calculated using the lateral radiographs of the goats at full flexion and full extension using the Cobb's method (Fig.1C-D). In detail, a straight line was drawn to connect the anterior and posterior corners of the superior vertebral body; the other line was drawn on the top of the inferior vertebral body; the Cobb angle was then formed by these two lines. Kyphotic angle was considered to be a positive value. Using quantitative measurement analysis software (DICOM), the segmental ROM can be calculated by the difference between full flexion and full extension angles of lumbar segments. The segmental ROM was measured three times by two blinded assessors with spine research experience and the mean value was determined. Segmental ROMs at the normal untreated controls were calculated as well. Interobserver reliability was assessed by intraclass correlation coefficient (ICC) which was calculated using a two-way random model and characterized by absolute agreement.

\subsection{Histological staining of the adjacent levels}

All the five goats were sacrificed by overdosed Pentobarbitone at 12 months postoperatively. The disc-transplanted segments were harvested en bloc along with the adjacent levels and fixed in $4 \%$ paraformaldehyde in phosphate-buffered saline for 7 days. The adjacent discs were separated and cut mid-sagittally using a band saw

(EXAKT 300CP Band System, Germany), and one half of them were decalcified, 
dehydrated, embedded in paraffin wax and finally cut into 5- $\mu$ m-thick sections and stained with hematoxylin and eosin (HE), Masson-trichrome (MT) and toluidine blue (TB).

\subsection{Statistical analysis}

All values were expressed as Median and Range. Non-parametric statistical analysis was performed to assess the DH and ROM in the allograft and in the adjacent levels after transplantation for 12 months by Kruskal-Wallis test. To compare the ROM of the disc-transplanted segment and the adjacent levels with those from the untreated control at postoperative 12 months, the Mann-Whitney test was used. A $P$ value $<0.05$ was considered statistically significant.

\section{Results}

All the IVD allografts were well seated and remained so throughout the follow-up period without subluxation or dislocation (Fig.2). No complication relating to the surgery or wound healing was found in the five goats after IVD allografting.

Immediately post operation, the median $\mathrm{DH}$ of the disc allograft was $2.8 \mathrm{~mm}$. This decreased slightly to $2.4 \mathrm{~mm}$ at $1 \mathrm{~m}$ post-operation which was not significant statistically $(P=0.117)$. Significant decrease of the DH started at $3 \mathrm{~m}$ postoperatively $(P<0.01)$ and at the final follow-up, the median DH of the allograft was $1.4 \mathrm{~mm}$

(Table 1). No notable decrease was recorded among the values of DH at 3, 6, 9 and 12 months postoperatively $(P=0.493)$. DH of the cranial disc exhibited no significant change throughout the entire observation period $(P=0.444)$; the median $\mathrm{DH}$ in the 
cranial segment ranged from $3.2 \mathrm{~mm}$ at $1 \mathrm{~m}$ post-operation to $3.0 \mathrm{~mm}$ at the final follow-up (Table 1). Similar finding was seen in the caudal level; the DH was not influenced by disc allografting which was well maintained without notable change (Table 1).

For the assessment of ROM data, the average of ICC was $0.90(95 \% \mathrm{CI}$ : 0.83-0.94) which indicated an excellent agreement. As shown in Table 2, the median ROM of the transplanted segments was $5.4^{\circ}$ at $1 \mathrm{~m}$ post-transplantation and at the final follow-up its value was $4.9^{\circ}$; no significant difference in the ROM of the disc-transplanted segment was seen during the whole observation period $(P=0.862)$. The ROM of the transplanted disc was comparable to that of the L4/L5 disc in the untreated control $\left(4.9^{\circ}, 3.1 \sim 9.2^{\circ}\right)$ at the 12-month follow-up $(P=0.834)$.Additionally, no obvious change in the ROM of the cranial segment was recorded $(P=0.943)$ after disc allografting for 12 months. At the last follow-up, the median segmental ROM of the cranial segment was $7.2^{\circ}$ which was comparable to that of the normal L3/L4 segment $(P=0.602)$. Similar finding was seen in the caudal level, the segmental ROM was not influenced by disc allografting. As shown in Table 2, the ROM at the caudal segment was comparable to that of the normal L5/L6 segment at postoperative 12 months $(P=0.076)$.

On gross examination, the architecture of the adjacent discs was well maintained at 12 months after disc transplantation (Fig. 3). No tearing or cleft formation was found. Normal gelatinous appearance was observed in the nucleus pulposus. The fibrocartilage lamellae without ruptured fibers of both the anterior and posterior 
annulus fibrosus could be clearly seen, and the border between the nucleus pulposus and annulus fibrosus tissue was well defined. Additionally, the thickness of the bony and cartilaginous endplates was uniform and normal.

\section{Discussion}

In our previous monkey study, DH of the disc allograft at 3 months decreased quickly to $73.1 \%$ of the original allograft height [17]. In the present study, a similar decrease occurred at 3 months after transplantation in goats. There are a number of possible reasons that may explain this loss of DH in the fresh-frozen allograft. First, it was previously determined that a proportion of disc cells will die after the cryopreservation of bovine and porcine IVD allografts [18, 19]. Second, during the process of fresh-frozen IVD allograft transplantation, osteotomies are performed on the adjacent vertebral bodies, rendering the IVD allograft in an ischemic state until the nutrient supply is reestablished. During this period, some of the disc cells die as they are deprived of nutrients, which in turn leads to disc degeneration [20-22].

The etiology of ASD after spine arthroplasty or arthrodesis remains unclear [10, 23]. The most commonly accepted postulations include abnormal biomechanical stress after fusion or disc replacements, natural history of degeneration in the adjacent discs, and disruption of anatomy during surgery [11]. Interestingly, ASD was not encountered after IVD allograft transplantation at 12 months in the large animals [17], and also in the human cervical spine at the 5 to 10 year follow ups $[4,5]$. During surgery, only one targeted level was exposed and no internal fixation was used, so that 
the soft tissue surrounding the adjacent segments was not disrupted. In the case of mal-positioned disc allografting in human cervical spine, relocation of the annulus fibrosus and preservation of nucleus were found after 5-year follow-up, indicating that biological remodeling may take place [4]. This similar biological activity was also seen in the goat lumbar spine, where the kinematic parameters of the segment with mal-positioned allograft transplantation became comparable to that of the normal spine after 6 months [6]. The anatomical and kinematical remodeling of the allograft thus may not have induced abnormal stresses in the adjacent segments. Therefore, disc allografting did not negatively affect the $\mathrm{DH}, \mathrm{ROM}$ and anatomy of the adjacent segments after 12-month follow-up.

We acknowledge that the goats used for disc transplantation were young and that the sample size was small. The small sample size may increase the opportunity of type II statistical error or result in lack of statistical power. Hence, in future study, a larger simple size should be considered. In a goat model of spinal fusion, a 6-month follow-up was enough to observe the development of ASD [24]. But whether a 12-month follow-up is sufficient enough for the ASD to develop after IVD allografting needs further investigation. Functional flexion-extension radiography is commonly used clinically for the assessment of vertebral stability and mobility. The second limitation in this study is that the full-flexion and -extension positions of the goats were made passively under general anaesthesia, although this method has been accepted also by other researchers $[6,14]$. The third limitation is that the goats are quadruped and do not reproduce the exact anatomy, loading and intradiscal pressures 
of the lumbar spine in the humans $[25,26]$. In large animals such as goats, sheep, pigs and dogs, loading of their lumbar spines are probably higher than that of human since much more muscle forces and passive tension are required to stabilize the horizontally aligned spine than the vertically one [26]; in addition, the in vivo intradiscal pressure in the ovine disc was reported to be much higher than that of the human disc [27]. Nevertheless, the goat model at present is the closest alternative short of the primates for fresh-frozen IVD allografting.

To conclude we have confirmed that fresh-frozen intervertebral disc transplantation could maintain the mobility of the functional spinal unit at a long term follow-up in a large animal model. Importantly, adjacent segment degeneration is not observed after this motion preservation technology, most likely because of the biological remodeling potential and restoration of near-normal segmental mobility.

\section{Acknowledgments}

This work was financially supported by the Research Grants Council of Hong Kong (773112) and the Tam Sai Kit Endowment Fund. We are very grateful to Mr. Jun-Ling Zeng from Nanfang Hospital (Guangzhou, China) for kind help in the animal experiments.

\section{References}

1. Borenstein D (2013) Mechanical low back pain--a rheumatologist's view. Nat Rev Rheumatol 9:643-653. doi: 10.1038/nrrheum.2013.133nrrheum.2013.133 [pii]

2. Chou R, Baisden J, Carragee EJ, Resnick DK, Shaffer WO, Loeser JD (2009) Surgery for low back pain: a review of the evidence for an American Pain Society 
Clinical Practice Guideline. Spine (Phila Pa 1976) 34:1094-1109. doi: 10.1097/BRS.0b013e3181a105fc

3. van den Eerenbeemt KD, Ostelo RW, van Royen BJ, Peul WC, van Tulder MW (2010) Total disc replacement surgery for symptomatic degenerative lumbar disc disease: a systematic review of the literature. Eur Spine J 19:1262-1280. doi: 10.1007/s00586-010-1445-3

4. Ruan D, He Q, Ding Y, Hou L, Li J, Luk KD (2007) Intervertebral disc transplantation in the treatment of degenerative spine disease: a preliminary study. Lancet 369:993-999. doi: S0140-6736(07)60496-6 [pii] 10.1016/S0140-6736(07)60496-6

5. Ding Y, Ruan DK, He Q, Hou LS, Lin JN, Cui HP (2013) Imaging Evaluation and Relative Significance in Cases of Cervical Disc Allografting: Radiographic Character Following Total Disc Transplantation. J Spinal Disord Tech. doi: 10.1097/BSD.0b013e318290fc41

6. Lam SK, Xiao J, Ruan D, Ding Y, Lu WW, Luk KD (2012) The effect of remodeling on the kinematics of the malpositioned disc allograft transplantation. Spine (Phila Pa 1976) 37:E357-366. doi: 10.1097/BRS.0b013e318232909d

7. Harrop JS, Youssef JA, Maltenfort M, Vorwald P, Jabbour P, Bono CM, Goldfarb N, Vaccaro AR, Hilibrand AS (2008) Lumbar adjacent segment degeneration and disease after arthrodesis and total disc arthroplasty. Spine (Phila Pa 1976) 33:1701-1707. doi: 10.1097/BRS.0b013e31817bb956 00007632-200807010-00016 [pii]

8. Ghiselli G, Wang JC, Bhatia NN, Hsu WK, Dawson EG (2004) Adjacent segment degeneration in the lumbar spine. J Bone Joint Surg Am 86-A:1497-1503

9. Hou Y, Liu Y, Yuan W, Wang X, Chen H, Yang L, Zhang Y (2014) Cervical kinematics and radiological changes after Discover artificial disc replacement versus fusion. Spine J 14:867-877. doi: 10.1016/j.spinee.2013.07.432

S1529-9430(13)01267-9 [pii]

10. Burkus JK, Traynelis VC, Haid RW, Jr., Mummaneni PV (2014) Clinical and radiographic analysis of an artificial cervical disc: 7-year follow-up from the Prestige prospective randomized controlled clinical trial: Clinical article. J Neurosurg Spine 21:516-528. doi: 10.3171/2014.6.SPINE13996

11. Helgeson MD, Bevevino AJ, Hilibrand AS (2013) Update on the evidence for adjacent segment degeneration and disease. Spine J 13:342-351. doi: 10.1016/j.spinee.2012.12.009S1529-9430(13)00070-3 [pii]

12. Lund T, Oxland TR (2011) Adjacent level disk disease--is it really a fusion disease? Orthop Clin North Am 42:529-541, viii. doi: 10.1016/j.ocl.2011.07.006 S0030-5898(11)00072-1 [pii]

13. Xiao J, Huang YC, Lam SK, Luk KD (2015) Surgical technique for lumbar intervertebral disc transplantation in a goat model. Eur Spine J 24:1951-1958. doi: 10.1007/s00586-014-3631-1

14. Gunzburg R, Szpalski M, Callary SA, Colloca CJ, Kosmopoulos V, Harrison D, Moore RJ (2009) Effect of a novel interspinous implant on lumbar spinal range of motion. Eur Spine J 18:696-703. doi: 10.1007/s00586-009-0890-3 
15. An HS, Takegami K, Kamada H, Nguyen CM, Thonar EJ, Singh K, Andersson GB, Masuda K (2005) Intradiscal administration of osteogenic protein-1 increases intervertebral disc height and proteoglycan content in the nucleus pulposus in normal adolescent rabbits. Spine (Phila Pa 1976) 30:25-31; discussion 31-22. doi: 00007632-200501010-00005 [pii]

16. Pfirrmann CW, Metzdorf A, Elfering A, Hodler J, Boos N (2006) Effect of aging and degeneration on disc volume and shape: A quantitative study in asymptomatic volunteers. J Orthop Res 24:1086-1094. doi: 10.1002/jor.20113

17. Luk KD, Ruan DK, Lu DS, Fei ZQ (2003) Fresh frozen intervertebral disc allografting in a bipedal animal model. Spine (Phila Pa 1976) 28:864-869; discussion 870. doi: 10.1097/01.BRS.0000058710.01729.29

18. Chan SC, Gantenbein-Ritter B, Leung VY, Chan D, Cheung KM, Ito K (2010) Cryopreserved intervertebral disc with injected bone marrow-derived stromal cells: a feasibility study using organ culture. Spine J 10:486-496. doi: 10.1016/j.spinee.2009.12.019S1529-9430(10)00003-3 [pii]

19. Chan SC, Lam S, Leung VY, Chan D, Luk KD, Cheung KM (2010) Minimizing cryopreservation-induced loss of disc cell activity for storage of whole intervertebral discs. Eur Cell Mater 19:273-283. doi: vol019a26 [pii]

20. Adams MA, Roughley PJ (2006) What is intervertebral disc degeneration, and what causes it? Spine 31:2151-2161

21. Grunhagen T, Shirazi-Adl A, Fairbank JC, Urban JP (2011) Intervertebral disk nutrition: a review of factors influencing concentrations of nutrients and metabolites. Orthop Clin North Am 42:465-477, vii. doi: S0030-5898(11)00076-9 [pii]

10.1016/j.ocl.2011.07.010

22. Huang YC, Urban JP, Luk KD (2014) Intervertebral disc regeneration: do nutrients lead the way? Nat Rev Rheumatol 10:561-566. doi: 10.1038/nrrheum.2014.91nrrheum.2014.91 [pii]

23. Luo J, Gong M, Huang S, Yu T, Zou X (2015) Incidence of adjacent segment degeneration in cervical disc arthroplasty versus anterior cervical decompression and fusion meta-analysis of prospective studies. Arch Orthop Trauma Surg 135:155-160. doi: 10.1007/s00402-014-2125-2

24. Hoogendoorn RJ, Helder MN, Wuisman PI, Bank RA, Everts VE, Smit TH (2008) Adjacent segment degeneration: observations in a goat spinal fusion study. Spine (Phila Pa 1976) 33:1337-1343. doi: 10.1097/BRS.0b013e318173438f 00007632-200805200-00010 [pii]

25. Smit TH (2002) The use of a quadruped as an in vivo model for the study of the spine - biomechanical considerations. Eur Spine J 11:137-144. doi: 10.1007/s005860100346

26. Alini M, Eisenstein SM, Ito K, Little C, Kettler AA, Masuda K, Melrose J, Ralphs J, Stokes I, Wilke HJ (2008) Are animal models useful for studying human disc disorders/degeneration? Eur Spine J 17:2-19. doi: 10.1007/s00586-007-0414-y

27. Reitmaier S, Schmidt H, Ihler R, Kocak T, Graf N, Ignatius A, Wilke HJ (2013) Preliminary investigations on intradiscal pressures during daily activities: an in vivo study using the merino sheep. PLoS One 8:e69610. doi: 
10.1371/journal.pone.0069610PONE-D-13-09319 [pii] 


\section{Figure legends}

Fig.1 (A-B) Methods for taking radiographs of the goats at full flexion and full extension. (C-D) Methods of Cobb's angle calculation and ROM measurement.

Fig.2 Serial follow-up radiographs of the lumbar spine after IVD allograft transplantation. The disc-transplanted segments (L4/L5) were shown in the red dotted rectangular area.

Fig.3 The structure of the adjacent levels was well preserved after disc transplantation for 12 months. Scale bar $=1 \mathrm{~mm}$. Abbreviations: A, anterior; P, posterior; AF, annulus fibrosus; NP, nucleus pulposus; CEP, cartilaginous endplate; HE, hematoxylin and eosin; MT, masson-trichrome; TB, toluidine blue.

Table 1 Change of the DHs at the allograft and the adjacent segments. After transplantation, $\mathrm{DH}$ of the disc allograft could be maintained for 1 month but was obviously decreased at 3 months postoperatively $(P<0.01)$; it was then preserved until completion of the follow-up $(P>0.05)$. IVD allografting did not change the $\mathrm{DH}$ of the cranial and caudal segments after 12 -month follow-up $(P>0.05)$. $* * P<0.01$, vs. DH of the IVD allograft immediately post operation. Abbreviation: DT, disc-transplanted.

Table 2 Motion preservation at the IVD-transplanted and the adjacent segments. The segmental ROM of the IVD-transplanted segment was well preserved, and no significant difference was found when compared with the normal untreated control at the final follow-up $(P>0.05)$.Similarly, ROM of the cranial and caudal segments was comparable to that of normal untreated control (L3/L4 and L5/L6) at the final follow-up $(P>0.05)$, respectively. Abbreviation: DT, disc-transplanted. 


\begin{tabular}{|c|c|c|c|}
\hline $\begin{array}{c}\text { Group } \\
\text { Time }\end{array}$ & $\begin{array}{c}\text { DT segment } \\
(\mathrm{L} 4 / \mathrm{L} 5, \mathrm{~mm})\end{array}$ & $\begin{array}{c}\text { Cranial segment } \\
(\mathrm{L} 3 / \mathrm{L} 4, \mathrm{~mm})\end{array}$ & $\begin{array}{c}\text { Caudal segment } \\
(\mathrm{L} 5 / \mathrm{L} 6, \mathrm{~mm})\end{array}$ \\
\hline $0 \mathrm{~m}$ & $2.8(2.3 \sim 3.4)$ & $3.2(2.6 \sim 3.6)$ & $3.6(3.1 \sim 4.1)$ \\
$1 \mathrm{~m}$ & $2.4(2.0 \sim 3.3)$ & $2.8(1.7 \sim 3.5)$ & $3.2(2.8 \sim 3.9)$ \\
$3 \mathrm{~m}$ & $2.0(1.4 \sim 2.1)^{* *}$ & $3.2(2.8 \sim 3.6)$ & $3.4(2.6 \sim 4.1)$ \\
$6 \mathrm{~m}$ & $1.5(1.1 \sim 2.6)^{* *}$ & $3.2(2.8 \sim 3.6)$ & $3.8(3.0 \sim 4.7)$ \\
$9 \mathrm{~m}$ & $1.5(0.8 \sim 2.5)^{* *}$ & $3.5(2.6 \sim 3.7)$ & $3.9(3.1 \sim 4.4)$ \\
$12 \mathrm{~m}$ & $1.4(0.5 \sim 2.1)^{* *}$ & $3.0(2.3 \sim 3.8)$ & $3.4(2.9 \sim 4.0)$ \\
\hline
\end{tabular}

Table 1 Change of the DH at the allograft and the adjacent segments

\begin{tabular}{|c|c|c|c|}
\hline $\begin{array}{c}\text { Group } \\
\text { Time }\end{array}$ & $\begin{array}{c}\text { DT segment } \\
\left(\mathrm{L} 4 / \mathrm{L} 5,^{\circ}\right)\end{array}$ & $\begin{array}{c}\text { Cranial segment } \\
\left(\mathrm{L} 3 / \mathrm{L} 4,^{\circ}\right)\end{array}$ & $\begin{array}{c}\text { Caudal segment } \\
\left(\mathrm{L} 5 / \mathrm{L} 6,{ }^{\circ}\right)\end{array}$ \\
\hline $1 \mathrm{~m}$ & $5.4(3.9 \sim 12)$ & $5.9(2.9 \sim 10.8)$ & $5.8(3.6 \sim 8.9)$ \\
$3 \mathrm{~m}$ & $4.4(2.8 \sim 7.1)$ & $5.3(4.4 \sim 11.8)$ & $9.1(2.1 \sim 10.2)$ \\
$6 \mathrm{~m}$ & $4.7(2.4 \sim 6.5)$ & $8.6(3.8 \sim 8.7)$ & $6.0(3.3 \sim 13.4)$ \\
$9 \mathrm{~m}$ & $6.7(2.7 \sim 6.5)$ & $6.9(2.3 \sim 9.1)$ & $6.4(0.6 \sim 14)$ \\
$12 \mathrm{~m}$ & $4.9(2.4 \sim 8.5)$ & $7.2(1.3 \sim 12.6)$ & $11(5.1 \sim 16.9)$ \\
\hline Untreated & $4.9(3.1 \sim 9.2)$ & $6.1(2.9 \sim 9.4)$ & $6.2(3.5 \sim 9.5)$ \\
control & & & \\
\hline
\end{tabular}

Table 2 Motion preservation in the IVD-transplanted and the adjacent segments 

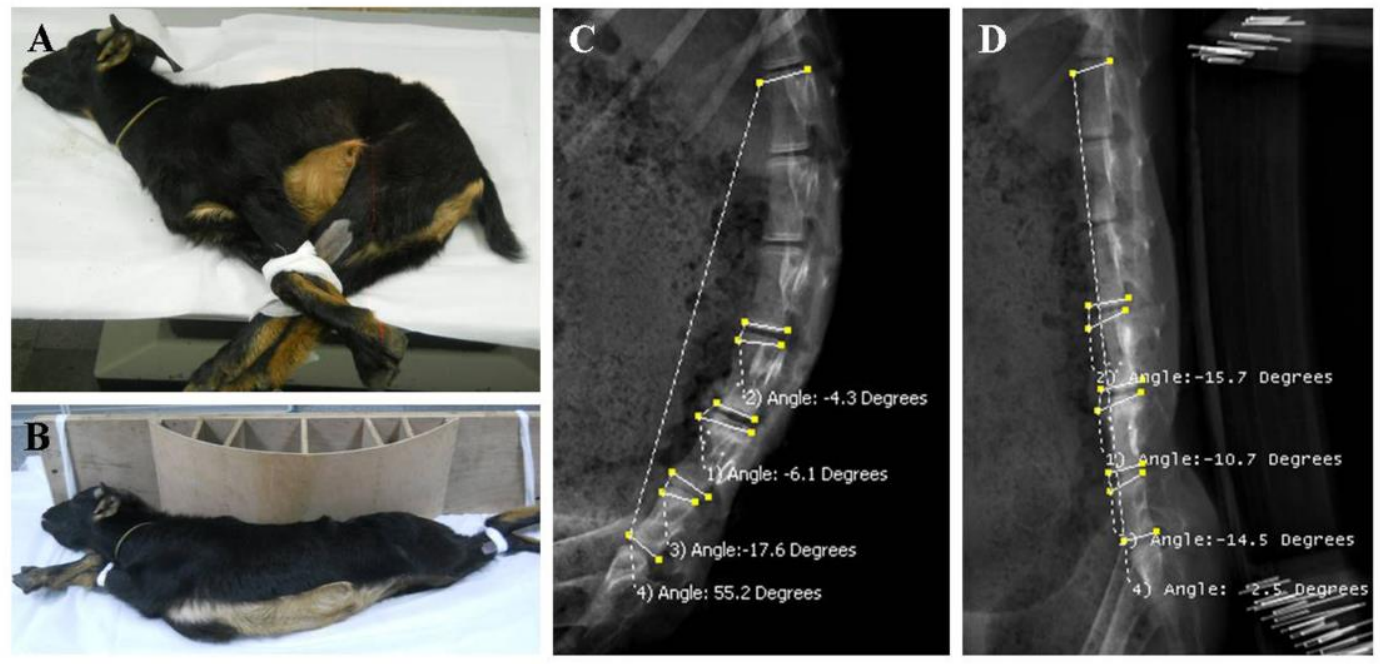


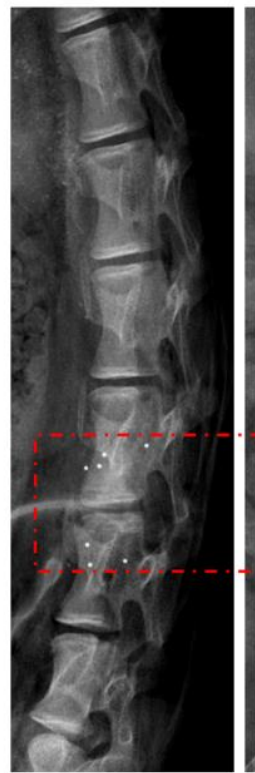

Immediate post-op

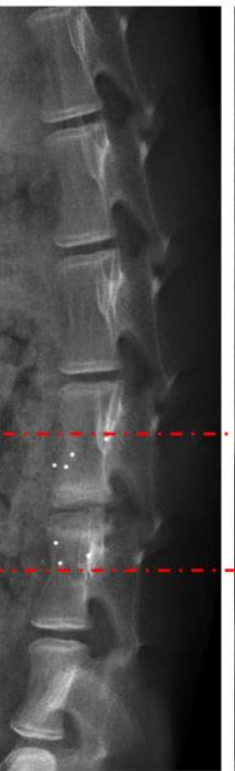

$1 \mathrm{~m}$ post-op

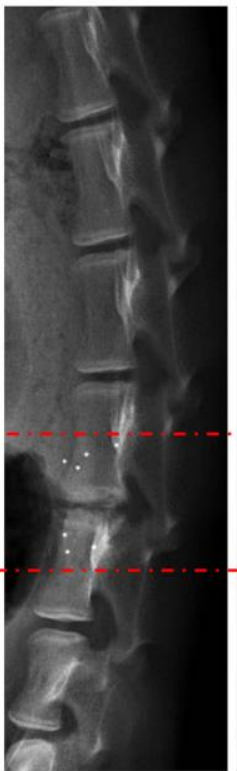

$3 m$ post-op

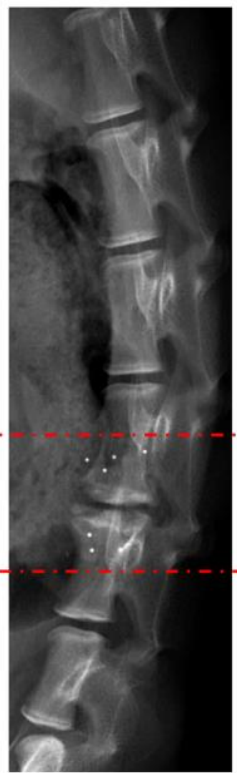

$6 \mathrm{~m}$ post-op

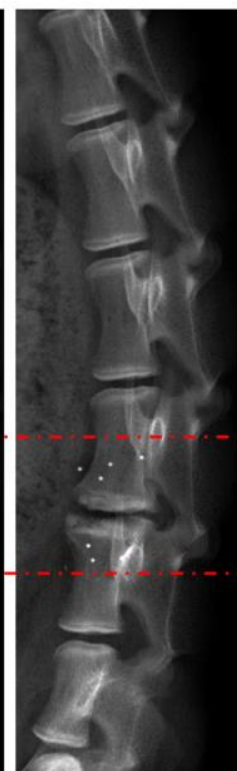

9m post-op

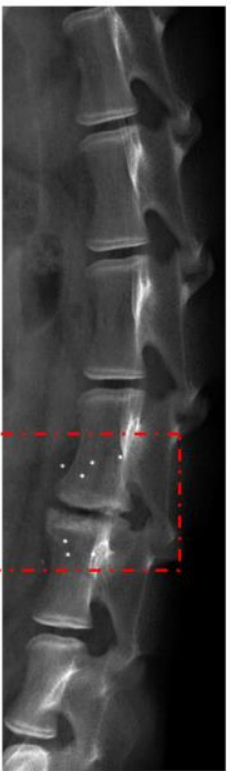

$12 \mathrm{~m}$ post-op 

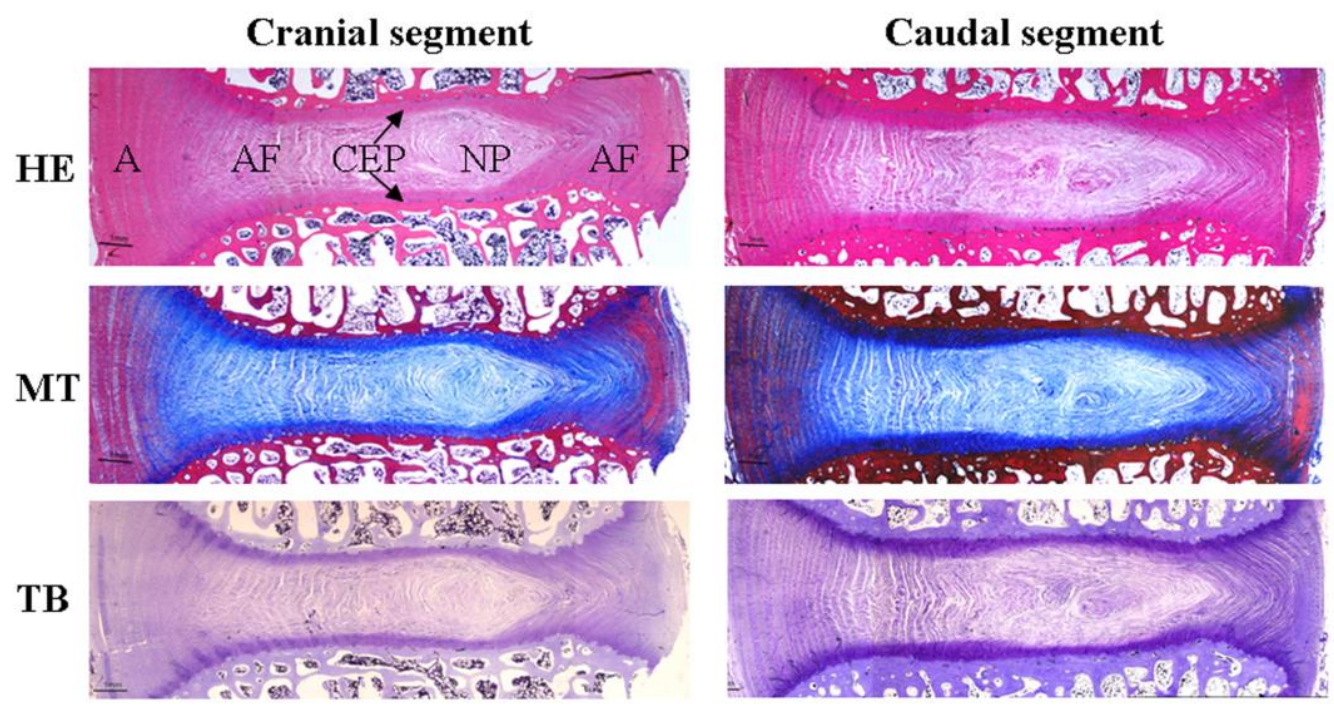